\begin{tabular}{|c|l|}
\hline Title & Theory of acoustic-phonon transmission in finite size superlattice systems \\
\hline Author(s) & Mizuno, Seiji; Tamura, Shin-ichiro \\
\hline Citation & $\begin{array}{l}\text { Physical Review B, 45(2), 734.741 } \\
\text { https://doi.org/40.1103/PhysRevB.45.734 }\end{array}$ \\
\hline Issue Date & 1992-01-01 \\
\hline Doc URL & http://hdl.handle.net/2115/5725 \\
\hline Rights & Copyright $\odot$ 1992 A merican Physical Society \\
\hline Type & article \\
\hline File Information & PRB45-2.pdf \\
\hline
\end{tabular}

Instructions for use 


\title{
Theory of acoustic-phonon transmission in finite-size superlattice systems
}

\author{
Seiji Mizuno and Shin-ichiro Tamura \\ Department of Engineering Science, Hokkaido University, Sapporo 060, Japan
}

(Received 19 August 1991)

\begin{abstract}
We develop a theory for acoustic-phonon transmission through a periodic superlattice with a finite number of periods. In particular, analytical expressions for the product of transfer matrices and phonon transmission rate are derived for the phonon propagation normal to the layer interfaces. The results are applied to the resonance phenomena of phonons in the triple-superlattice structure, i.e., the stack $A B A$ of the periodic superlattices $A$ and $B$. The phonon transmission rate and the resonance condition in this structure are also derived analytically. As a result, we show that the phonons in a frequency gap of the $A$ superlattice can be transmitted significantly through the $A B A$ superlattice structure when they satisfy the resonance condition. This happens even if the period of the $A$ superlattice is infinity.
\end{abstract}

\section{INTRODUCTION}

Acoustic phonon propagation in synthetic superlattice systems with various stacking order, such as periodic, quasiperiodic, and random superlattices, has been investigated both theoretically ${ }^{1}$ and experimentally. ${ }^{2}$ For phonons propagating in a periodic superlattice Bragg reflection occurs when the periodicity matches with their wavelength, yielding frequency gaps (i.e., stop bands) in the phonon dispersion relation. ${ }^{3}$ Thus, the periodic superlattice exhibits a filtering action on phonons in the stop bands. ${ }^{2}$ By utilizing this property of phonons in the superlattice, we can basically realize an elastic multilayered system which may selectively transmit or reflect the phonons in some frequency window. This suggests the potential of designing a variety of phonon optics ${ }^{4}$ devices by using single- or multiple-superlattice structures.

Recently, the numerical study on the transmission of phonons in the simplest multiple-superlattice system, where a periodic $B$ superlattice is sandwiched by different periodic $A$ superlattices, i.e., $A B A$ superlattice structure, has been reported. ${ }^{5}$ In this system, the phonons in a stop band of the $A$ superlattice ( $A$ stop band) can be transmitted almost perfectly through the $A B A$ system when their frequency satisfies a certain resonance condition. This is analogous to the resonant tunneling of electrons in multiple-quantum-well systems. ${ }^{6}$ So far, almost all of the phonon transmission calculation has been carried out numerically based on the transfer-matrix method. ${ }^{7}$ This is partly because in a real superlattice system the number of periods is finite and the perfect periodicity, valid in an infinite system, is absent. However, analytic expressions for the transmission rate in finite size systems are desirable for studying the characteristic behaviors of phonons in a complicated superlattice structure.

In the present study, we derive the general expression for the phonon transmission rate in the finite-size superlattices by calculating the products of transfer matrices analytically. The results are applied to the phonon transmission in an $\boldsymbol{A B} \boldsymbol{A}$ multisuperlattice system, and we discuss the resonance condition and the enhancements in phonon transmission in this system.

The outline of this paper is as follows. In Sec. II, we develop the mathematical analysis of the transfer matrix and the transmission rate in the periodic superlattice with a finite number of periods. The numerical examples of the phonon transmission in the single- and doublesuperlattice structures based on our formula are also presented. In Sec. III, we discuss the transmission of acoustic phonons in the $A B A$ multisuperlattice system. Specifically, the phonon transmission rate and resonance condition are derived by using the analytic expression for the transmission rate in single periodic superlattices given in Sec. II. The sharp enhancements in transmission are predicted at phonon frequencies satisfying the resonance condition. In Sec. IV, a summary and conclusions are given.

\section{MATHEMATICAL ANALYSIS OF TRANSFER MATRIX AND PHONON TRANSMISSION RATE}

We study a periodic superlattice with a finite number of periods. A schematic picture of this system is shown in Fig. 1. Here, we assume that the superlattice consists of a periodic sequence of alternate stacking of $A_{1}$ and $A_{2}$ constituent layers. The thicknesses of the $A_{1}$ and $A_{2}$ layers are denoted by $d_{A_{1}}$ and $d_{A_{2}}$, respectively, and $D_{A}$ ( $=d_{A_{1}}+d_{A_{2}}$ ) is the thickness of the unit period of this system.

We consider the case where the phonon wave vector is parallel to the growth direction ( $z$ direction). For this propagation configuration all three phonon modes are decoupled from each other if the interfaces are a mirrorsymmetry plane. We consider such a simple situation and treat only one mode of phonons. In the present study, we adopt the continuum model for the lattice vibration, which is valid for sub- $\mathbf{T H z}$ acoustic phonons in most of the semiconductor superlattices. The solution to the one-dimensional wave equation for this elastic continuum is expressed in terms of a linear combination of the transmitted and reflected waves: 


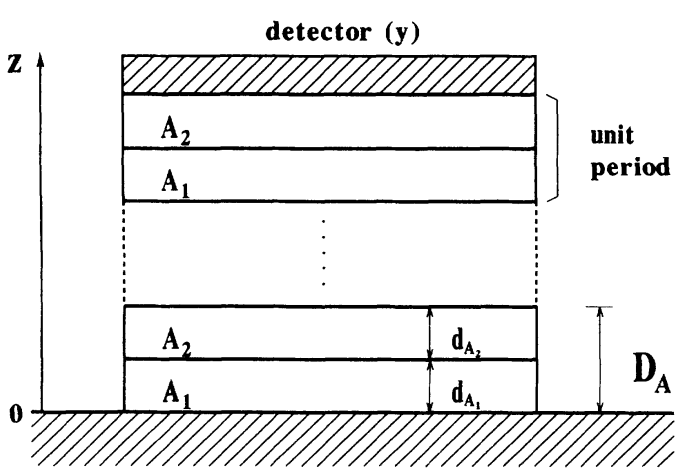

substrate $(\mathbf{x})$

FIG. 1. Schematic picture of the periodic superlattice with the $N$ periods. The superlattice consists of a sequence of alternate stacking of $A_{1}$ and $A_{2}$ constituent layers. The thicknesses of the $A_{1}$ and $A_{2}$ layers are denoted by $d_{A_{1}}$ and $d_{A_{2}}$, respectively, and $D_{A}\left(=d_{A_{1}}+d_{A_{2}}\right)$ represents the thickness of the unit period.

$$
U_{i}(z)=c_{i}^{t} e^{i k_{i} z}+c_{i}^{r} e^{-i k_{i} z}, \quad i=A_{1}, A_{2} .
$$

Here, $i$ is an index specifying constituent layers, $c_{i}^{t}$ and $c_{i}^{r}$ are the amplitudes of the transmitted and reflected waves, respectively, and $k_{i}$ is the wave number. The stress associated with the lattice displacement $U_{i}$ is expressed as

$$
S_{i}(z)=i \omega Z_{i}\left(c_{i}^{t} e^{i k_{i} z}-c_{i}^{r} e^{-i k_{i} z}\right),
$$

where $Z_{i}\left(=\rho_{i} v_{i}\right)$ is the acoustic impedance with the mass density $\rho_{i}$ and the sound velocity $v_{i}$, and $\omega=k_{i} v_{i}$ is the angular frequency.

The lattice displacement $U_{i}(z)$ and stress $S_{i}(z)$ should be continuous at the interfaces of adjacent layers. To write down these boundary conditions, it is convenient to introduce a two-component vector

$$
\mathbf{W}_{i}(z)=\left[\begin{array}{l}
U_{i}(z) \\
S_{i}(z)
\end{array}\right] .
$$

Let us consider the first layer $A_{1}$, sandwiched by the substrate denoted by $x$ and the second layer $A_{2}$ (see Fig. 1). The boundary conditions require

$$
\mathbf{W}_{A_{1}}(0)=\mathbf{W}_{x}(0)
$$

and

$$
\mathbf{W}_{A_{1}}\left(d_{A_{1}}\right)=\mathbf{W}_{A_{2}}\left(d_{A_{1}}\right) .
$$

By the definitions (1) and (2), $\mathbf{W}_{i}(z)$ can be written as

$$
\mathbf{W}_{i}(z)=\underline{h}_{i}(z) \mathbf{C}_{i}
$$

where

$$
\underline{h}_{i}(z)=\left[\begin{array}{cc}
e^{i k_{i} z} & e^{-i k_{i} z} \\
i \omega Z_{i} e^{i k_{i} z} & -i \omega Z_{i} e^{-i k_{i} z}
\end{array}\right],
$$

$$
\mathrm{C}_{i}=\left(\begin{array}{c}
c_{i}^{t} \\
c_{i}^{r}
\end{array}\right)
$$

Using Eq. (6), we can combine Eqs. (4) and (5) and get

$$
\mathbf{W}_{A_{2}}\left(d_{A_{1}}\right)=\underline{t}_{A_{1}}\left(d_{A_{1}}\right) \mathbf{W}_{x}(0),
$$

where

$$
\underline{t}_{A_{1}}\left(d_{A_{1}}\right)=\underline{h}_{A_{1}}\left(d_{A_{1}}\right)\left[\underline{h}_{A_{1}}(0)\right]^{-1} .
$$

This means that $\mathrm{W}$ changes to $\underline{t}_{A_{1}} \mathrm{~W}$ after the propagation of a wave through the first layer. An explicit expression for matrix $\underline{t}_{A_{1}}$ is

$$
\underline{t}_{A_{1}}\left(d_{A_{1}}\right)=\left(\begin{array}{cc}
\cos \alpha_{1} & \frac{1}{\omega Z_{A_{1}}} \sin \alpha_{1} \\
-\omega Z_{A_{1}} \sin \alpha_{1} & \cos \alpha_{1}
\end{array}\right],
$$

where $\alpha_{1}=k_{A_{1}} d_{A_{1}}$. Similarly, we can define a $2 \times 2$ matrix $\underline{t}_{A_{2}}$ for the $A_{2}$ layer. Using $\underline{t}_{A_{1}}$ and $\underline{t}_{A_{2}}$, we can now relate W's right before and after the unit period consisting of $A_{1}$ and $A_{2}$ layers,

$$
\mathrm{W}_{A_{1}}\left(D_{A}\right)=\underline{T}_{A} \mathrm{~W}_{A_{1}}(0) \text {. }
$$

The transfer matrix $\underline{T}_{A}$ is given by

$$
\underline{T}_{A}=\underline{t}_{A_{2}} \underline{t}_{A_{1}} \equiv\left(\begin{array}{cc}
\lambda_{A} & \frac{1}{\omega Z_{A_{1}}} \sigma_{A} \\
\omega Z_{A_{1}} \zeta_{A} & \mu_{A}
\end{array}\right),
$$

where

$$
\begin{aligned}
& \lambda_{A}=\cos \alpha_{1} \cos \alpha_{2}-\frac{Z_{A_{1}}}{Z_{A_{2}}} \sin \alpha_{1} \sin \alpha_{2}, \\
& \sigma_{A}=\sin \alpha_{1} \cos \alpha_{2}+\frac{Z_{A_{1}}}{Z_{A_{2}}} \cos \alpha_{1} \sin \alpha_{2}, \\
& \zeta_{A}=-\sin \alpha_{1} \cos \alpha_{2}-\frac{Z_{A_{2}}}{Z_{A_{1}}} \cos \alpha_{1} \sin \alpha_{2}, \\
& \mu_{A}=\cos \alpha_{1} \cos \alpha_{2}-\frac{Z_{A_{2}}}{Z_{A_{1}}} \sin \alpha_{1} \sin \alpha_{2},
\end{aligned}
$$

with $\alpha_{2}=k_{A_{2}} d_{A_{2}}$. We note that

$$
\operatorname{det}\left(\underline{T}_{A}\right)=\lambda_{A} \mu_{A}-\sigma_{A} \zeta_{A}=1 \text {. }
$$

For the superlattice with $N$ periods, we have to calculate $\left(\underline{T}_{A}\right)^{N} \equiv \underline{T}_{A}(N)$. To obtain the analytical expression of $\underline{T}_{A}(N)$, we first transform $\underline{T}_{A}$ into the diagonal matrix $\widetilde{T}_{A}$ :

$$
\widetilde{T}_{A}=\underline{S}^{-1} \underline{T}_{A} \underline{S}=\left(\begin{array}{ll}
\epsilon_{1} & 0 \\
0 & \epsilon_{2}
\end{array}\right),
$$

where $\epsilon_{1}$ and $\epsilon_{2}$ are the solutions of the equation

$$
\epsilon^{2}-\left(\mu_{A}+\lambda_{A}\right) \epsilon+1=0 \text {. }
$$

Solving Eq. (20), we have the diagonal elements of $\widetilde{T}_{A}$ : 
$\epsilon_{1,2}=\left\{\begin{array}{l}\frac{\mu_{A}+\lambda_{A}}{2} \pm i\left[1-\left[\frac{\mu_{A}+\lambda_{A}}{2}\right]^{2}\right]^{1 / 2} \text { for }\left|\frac{\mu_{A}+\lambda_{A}}{2}\right| \leq 1 \\ \frac{\mu_{A}+\lambda_{A}}{2} \pm\left[\left[\frac{\mu_{A}+\lambda_{A}}{2}\right]^{2}-1\right]^{1 / 2} \text { for }\left|\frac{\mu_{A}+\lambda_{A}}{2}\right|>1\end{array}\right.$

We explicitly express the transfer matrix $\underline{T}_{A}(N)$ for the following three cases.

(a) $\left|\left(\mu_{A}+\lambda_{A}\right) / 2\right| \leq 1$. In the frequency range satisfying this condition, we define $\theta_{A}$ by

$$
\cos \theta_{A} \equiv \frac{\mu_{A}+\lambda_{A}}{2} \text {. }
$$

Hence, $\epsilon_{1,2}$ can be written as

$$
\epsilon_{1,2}=\cos \theta_{A} \pm i \sin \theta_{A}=e^{ \pm i \theta_{A}}
$$

and the corresponding matrix $\underline{S}$ is given by

$$
\underline{S}=\left[\begin{array}{cc}
-\sigma_{A} & -\sigma_{A} \\
\lambda_{A}-e^{i \theta_{A}} & \lambda_{A}-e^{-i \theta_{A}}
\end{array}\right) .
$$

With the use of Eqs. (19) and (24), $\underline{T}_{A}(N)$ is calculated as

$$
\begin{aligned}
\underline{T}_{A}(N) & \equiv\left(\underline{T}_{A}\right)^{N} \\
& =\underline{S}\left(\underline{\widetilde{T}}_{A}\right)^{N} \underline{S}^{-1} \\
& =\left(\begin{array}{cc}
\frac{\lambda \lambda_{A}-\mu_{A}}{2} S_{A}(N)+C_{A}(N) & \frac{1}{\omega Z_{A_{1}}} \sigma_{A} S_{A}(N) \\
\omega Z_{A_{1}} \zeta_{A} S_{A}(N) & -\frac{\lambda_{A}-\mu_{A}}{2} S_{A}(N)+C_{A}(N)
\end{array}\right),
\end{aligned}
$$

where

$$
\begin{aligned}
& S_{A}(N) \equiv \frac{\sin \left(N \theta_{A}\right)}{\sin \theta_{A}}, \\
& C_{A}(N) \equiv \cos \left(N \theta_{A}\right) .
\end{aligned}
$$

Thus, the elements of $\underline{T}_{A}(N)$ has an oscillatory behavior.

(b) $\left(\mu_{A}+\lambda_{A}\right) / 2>1$. In this case, we define $\theta_{A}$ by

$$
\cosh \theta_{A} \equiv \frac{\mu_{A}+\lambda_{A}}{2} \text {. }
$$

Then, $\epsilon_{1,2}$ can be written as

$$
\epsilon_{1,2}=\cosh \theta_{A} \pm \sinh \theta_{A}=e^{ \pm \theta_{A}}
$$

and $\underline{T}_{A}(N)$ has the same form as Eq. (25), but with

$$
\begin{aligned}
& S_{A}(N) \equiv \frac{\sinh \left(N \theta_{A}\right)}{\sinh \theta_{A}}, \\
& C_{A}(N) \equiv \cosh \left(N \theta_{A}\right) .
\end{aligned}
$$

Thus, the elements of $\underline{T}_{A}(N)$ has an exponential behavior in this case.

(c) $\left(\mu_{A}+\lambda_{A}\right) / 2<-1$. In this case, we define $\theta_{A}$ by

$$
\cosh \theta_{A} \equiv-\frac{\mu_{A}+\lambda_{A}}{2} \text {. }
$$

Then, $\epsilon_{1,2}$ can be written as

$$
\epsilon_{1,2}=-\cosh \theta_{A} \mp \sinh \theta_{A}=-e^{ \pm \theta_{A}},
$$

and $\underline{T}_{A}(N)$ has the same form as Eq. (25), but with

$$
S_{A}(N) \equiv(-1)^{N+1} \frac{\sinh \left(N \theta_{A}\right)}{\sinh \theta_{A}},
$$

$$
C_{A}(N) \equiv(-1)^{N} \cosh \left(N \theta_{A}\right) .
$$

The elements of $\underline{T}_{A}(N)$ has an exponential behavior, as in case (b).

Next, we calculate the phonon transmission rate by using the above analytical expressions for the transfer matrix. In a typical transmission experiment of phonons through a superlattice, a thin-film phonon detector (denoted by $y$ ) is deposited on top of the superlattice grown on a substrate $x$. High-frequency phonons are excited at the other face of the substrate by using a superconducting tunnel junction or by a laser excitation. Thus both the incident and reflected phonons exist in the substrate but there are only transmitted phonons in the detector layer. Hence for an incident phonon with unit displacement amplitude, we have

$$
\mathbf{W}_{x}(z)=\underline{h}_{x}(z)\left(\begin{array}{c}
1 \\
c_{x}^{r}
\end{array}\right)
$$

in the substrate and

$$
\mathbf{W}_{y}(z)=\underline{h}_{y}(z)\left(\begin{array}{l}
c_{y}^{t} \\
0
\end{array}\right)
$$

in the detector layer. The amplitudes of reflection $c_{x}^{r}$ and transmission $c_{y}^{t}$ are determined by the continuity condition for $\mathbf{W}$ at each layer interface. Using the transfer ma$\operatorname{trix} \underline{T}_{A}(N)$, this condition yields

$$
\mathbf{W}_{y}\left(N D_{A}\right)=\underline{T}_{A}(N) \mathbf{W}_{x}(0)
$$

For simplicity, we write the elements of $\underline{T}_{A}(N)$ as 


$$
\underline{T}_{A}(N)=\left(\begin{array}{cc}
a & \frac{1}{\omega Z_{A_{1}}} b \\
\omega Z_{A_{1}} c & d
\end{array}\right),
$$

where

$$
\begin{aligned}
a & =\frac{\lambda_{A}-\mu_{A}}{2} S_{A}(N)+C_{A}(N), \\
b & =\sigma_{A} S_{A}(N), \\
c & =\zeta_{A} S_{A}(N), \\
d & =-\frac{\lambda_{A}-\mu_{A}}{2} S_{A}(N)+C_{A}(N) .
\end{aligned}
$$

Accordingly, from Eqs. (36), (37), and (38), we find

$$
\begin{aligned}
& c_{y}^{t}=\frac{2 i}{\left(\frac{Z_{y}}{Z_{A_{1}}} b-\frac{Z_{A_{1}}}{Z_{x}} c\right)+i\left(d+\frac{Z_{y}}{Z_{x}} a\right)} e^{-i k_{y} N D}, \\
& c_{x}^{r}=\frac{\left(\frac{Z_{y}}{Z_{A_{1}}} b+\frac{Z_{A_{1}}}{Z_{x}} c\right)+i\left(d-\frac{Z_{y}}{Z_{x}} a\right)}{\left(\frac{Z_{y}}{Z_{A_{1}}} b-\frac{Z_{A_{1}}}{Z_{x}} c\right)+i\left(d+\frac{Z_{y}}{Z_{x}} a\right)} .
\end{aligned}
$$

The energy transmission rate $T$ and reflection rate $R$ are defined by

$$
\begin{aligned}
T & \equiv \frac{Z_{y}}{Z_{x}}\left|c_{y}^{t}\right|^{2}, \\
R & \equiv\left|c_{x}^{r}\right|^{2} .
\end{aligned}
$$

From Eqs. (44) and (45), we obtain

$$
\begin{aligned}
& T=\frac{4 \frac{Z_{y}}{Z_{x}}}{\left[\frac{Z_{y}}{Z_{A_{1}}} b-\frac{Z_{A_{1}}}{Z_{x}} c\right)^{2}+\left(d+\frac{Z_{y}}{Z_{x}} a\right)^{2}}, \\
& R=\frac{\left[\frac{Z_{y}}{Z_{A_{1}}} b+\frac{Z_{A_{1}}}{Z_{x}} c\right)^{2}+\left(d-\frac{Z_{y}}{Z_{x}} a\right)^{2}}{\left[\frac{Z_{y}}{Z_{A_{1}}} b-\frac{Z_{A_{1}}}{Z_{x}} c\right)^{2}+\left(d+\frac{Z_{y}}{Z_{x}} a\right)^{2}} .
\end{aligned}
$$

It can be easily seen that

$$
T+R=1 .
$$

From the expression (48), we find the following results. In cases (b) and (c), i.e., $\left|\left(\mu_{A}+\lambda_{A}\right) / 2\right|>1$, all the matrix elements of $\underline{T}_{A}(N)$ increase exponentially $\left(\sim e^{N \theta_{A}}\right)$ for large $N$ [see Eqs. (30), (31), (34), and (35)]. Therefore, the transmission rate decreases exponentially with increasing period $N$. Thus, the superlattice exhibits a strong filtering action on phonons with frequencies satisfying the condition $\left|\left(\mu_{A}+\lambda_{A}\right) / 2\right|>1$. These phonons correspond to those in the forbidden gaps (stop bands) of the ideal periodic superlattice with the infinite number of periods. On the other hand, in case (a), the matrix elements have the oscillatory behavior [cf., Eqs. (25)-(27)]. Therefore, the transmission rate is finite and the phonons can be transmitted through the superlattice. These phonons correspond to those in the allowed bands in the ideal
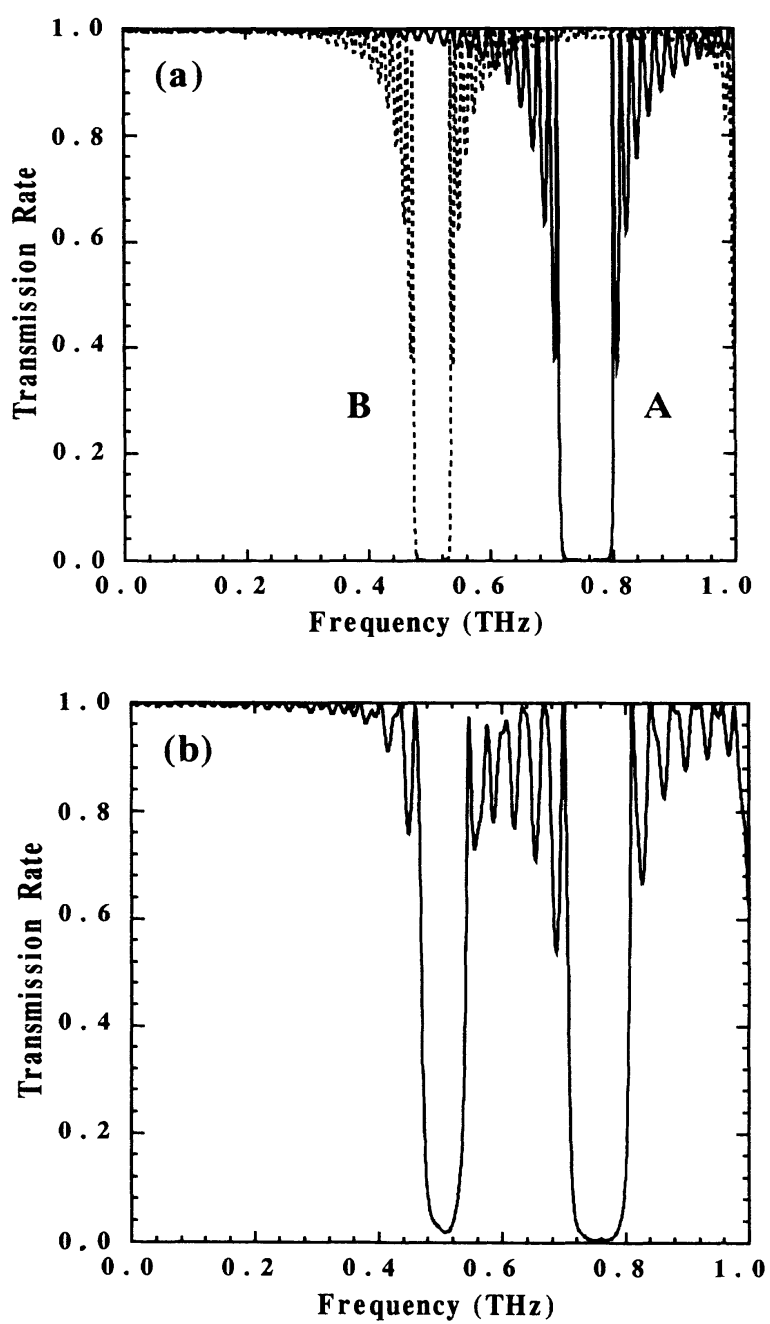

FIG. 2. Frequency dependence of the L-phonon transmission rates in (100) GaAs/AlAs superlattices: (a) the pure $A$-type superlattice (solid line) and the pure $B$-type superlattice (dotted line) $(N=M=35)$; (b) the $A B$ superlattice structure $(N=20$, $M=15)$. The assumed unit periods of $A$ and $B$ superlattices consist of ( $6 \mathrm{ML} \mathrm{AlAs}) /(6 \mathrm{ML} \mathrm{GaAs})$ and $(9 \mathrm{ML} \mathrm{AlAs}) /(9 \mathrm{ML}$ GaAs), respectively. The parameters used are as follows. The thickness of one monolayer (ML) is $2.83 \AA$ in the [100] direction for both GaAs $\left(=A_{2}=B_{2}\right)$ and AlAs $\left(=A_{1}=B_{1}\right)$. The mass density and longitudinal sound velocity are $5.36 \mathrm{~g} / \mathrm{cm}^{3}$ and 4.71 $\mathrm{km} / \mathrm{s}$ for GaAs, and $3.76 \mathrm{~g} / \mathrm{cm}^{3}$ and $5.65 \mathrm{~km} / \mathrm{s}$ for AlAs (i.e., $\rho_{A_{1}}=\rho_{B_{1}}=3.76 \mathrm{~g} / \mathrm{cm}^{3}, \rho_{A_{2}}=\rho_{B_{2}}=5.36 \mathrm{~g} / \mathrm{cm}^{3}, v_{A_{1}}=v_{B_{1}}=5.65$ $\mathrm{km} / \mathrm{s}, v_{A_{2}}=v_{B_{2}}=4.71 \mathrm{~km} / \mathrm{s}$ ). 
periodic superlattice with the infinite number of periods.

As a numerical example, we choose a (100) GaAs/AlAs superlattice and consider two periodic superlattices $A$ and $B$, whose unit periods consist of (6 ML AlAs)/(6 ML GaAs) and (9 ML AlAs)/(9 ML GaAs), respectively. The number of periods in the $A(B)$ superlattice is $N=35(M=35)$. In Fig. 2(a), we show the frequency dependence of the longitudinal- (L-) phonon transmission rates in these periodic superlattices calculated from Eqs. (39) and (48). We find a dip in the given frequency range ( 0 to $1 \mathrm{THz}$ ) for both the $A$-type and $B$-type superlattices. These dips are due to Bragg reflections of $\mathrm{L}$ phonons in each periodic superlattice. The frequencies at the center of these dips predicted by the first-order Bragg conditions $^{7}$ are 756 and $504 \mathrm{GHz}$ for the $A$-type and $B$ type superlattices. These values are in agreement with the frequencies at the center of the dips in Fig. 2(a).

We can also study the phonon transmission in the multisuperlattice systems based on the above expressions for the transfer matrix and the transmission rate in a periodic superlattice. For example, we consider the $A B$ superlattice system, where the periodic superlattice $A$ is stacked on another periodic superlattice $B$. The relevant transfer matrix in this double-superlattice structure is still $2 \times 2$ and given by the product of the matrix $\underline{T}_{A}(N)$ [Eq. (39)] and the similar matrix $\underline{T}_{B}(M)$ for superlattice $B$, which is obtained by replacing the symbols $A$ and $N$ with $B$ and $M$. Substituting the components of the product $\underline{T}_{A}(M) \underline{T}_{B}(N)$ into Eq. (48) we can obtain the phonon transmission rate in the $A B$ superlattice system. For the numerical example, the same $A$ and $B$ superlattices assumed above (but with $M=20$ and $N=15$ ) are stacked.

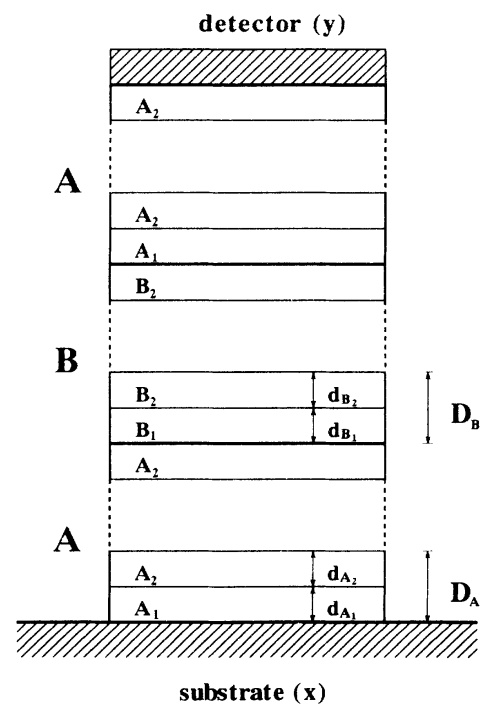

FIG. 3. Schematic picture of the $A B A$ triple superlattice. The superlattice $A(B)$ consists of a sequence of alternate stacking of $A_{1}$ and $A_{2}\left(B_{1}\right.$ and $\left.B_{2}\right)$ layers. The thicknesses of the $A_{1}$ and $A_{2}\left(B_{1}\right.$ and $\left.B_{2}\right)$ layers are denoted by $d_{A_{1}}$ and $d_{A_{2}}\left(d_{B_{1}}\right.$ and $\left.d_{B_{2}}\right)$, respectively, and $D_{A}=d_{A_{1}}+d_{A_{2}} \quad\left(D_{B}=d_{B_{1}}+d_{B_{2}}\right)$ represents the length of a period of $A(B)$ superlattice. The numbers of the periodicity in $A$ and $B$ superlattices are $N$ and $M$, respectively.
The transmission rate versus frequency in this system is shown in Fig. 2(b). Two large dips in the same frequency range as in Fig. 2(a) are found. Comparing with Fig. 2(a), these dips can be well identified with the dips in the pure $A$-type and $B$-type superlattices. Therefore, the main features of the transmission rate in the $A B$ superlattice can be explained in terms of those in the pure $A$-type and $B$-type superlattices. No prominent structure that is not seen in the pure $A$-type and $B$-type superlattices arises in the double-superlattice system.

\section{III. $A B A$ MULTIPLE-SUPERLATTICE SYSTEM}

In this section, we apply the analytical expression for the transfer matrix given in Sec. II to a triple-superlattice structure. The system we consider consists of periodic superlattices $A, B$, and $A$ grown on a substrate. The schematic picture is shown in Fig. 3. In this multiplesuperlattice system the sharp structures in phonon transmission not attributable to the original pure $A$ and $B$ superlattices can be seen.

For phonons propagating through this $A B A$ system the relevant transfer matrix is

$$
\begin{aligned}
\underline{T}_{A B A} & \equiv\left(T_{A}\right)^{N}\left(\underline{T}_{B}\right)^{M}\left(\underline{T}_{A}\right)^{N} \\
& =\underline{T}_{A}(N) \underline{T}_{B}(M) \underline{T}_{A}(N) .
\end{aligned}
$$

The calculation of the matrix product of Eq. (51) is straightforward. The explicit expression for $T_{A B A}$ is, however, lengthy and we have given in Appendix $A$ the matrix elements of $\underline{T}_{A B A}$ for

$$
\left|\frac{\mu_{A}+\lambda_{A}}{2}\right|>1 \text { and }\left|\frac{\mu_{B}+\lambda_{B}}{2}\right| \leq 1 \text {. }
$$

The substitution of these matrix elements to Eq. (48) gives the phonon transmission rate in the $A B A$ superlattice structure.

Figure 4 exhibits the calculated frequency dependence

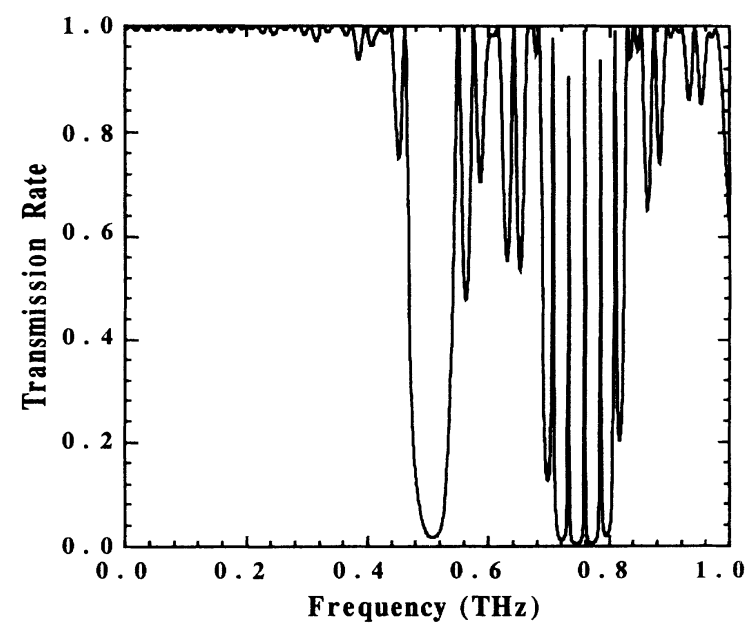

FIG. 4. Frequency dependence of the L-phonon transmission rate in the $A B A$ triple superlattice. The $A$ and $B$ superlattices are the same as for Fig. 2 but with $N=10$ and $M=15$. 
of phonon transmission in the $A B A$ structure where $A$ and $B$ superlattices are composed of 10 and 15 periods $(N=10, M=15)$ of GaAs/AlAs multilayers. (For simplicity, we assume that the substrate and the detector are the same materials, i.e., $x=y$.) The most noticeable feature is the appearance of three sharp enhancements in transmission in the dip $(0.716-0.797 \mathrm{THz})$ originating from the Bragg reflection in the $A$-type superlattice. In order to see the details in the former structure, the transmission rate in the vicinity of the $A$-stop band is enlarged in Fig. 5(a). These enhancements in transmission correspond to the resonant transmission, and their origin has been discussed only qualitatively in the previous work. ${ }^{5}$

Here, we discuss these resonant transmission quantitatively based on the analytical expression for the transfer matrix derived in Sec. II. We consider the phonons with a frequency satisfying the condition (52); that is, the assumed frequency is in a stop band of the pure $A$-type superlattice but in an allowed band of the pure $B$-type superlattice. Therefore, the transfer matrices $\underline{T}_{A}(N)$ and $\underline{T}_{B}(M)$ for this frequency are given by

$$
\begin{aligned}
& \underline{T}_{A}(N)=\left(\begin{array}{cc}
\frac{\lambda_{A}-\mu_{A}}{2} S_{A}(N)+C_{A}(N) & \frac{1}{\omega Z_{A_{1}}} \sigma_{A} S_{A}(N) \\
\omega Z_{A_{1}} \zeta_{A} S_{A}(N) & -\frac{\lambda_{A}-\mu_{A}}{2} S_{A}(N)+C_{A}(N)
\end{array}\right), \\
& \underline{T}_{B}(M)=\left(\begin{array}{cc}
\frac{\lambda_{B}-\mu_{B}}{2} S_{B}(M)+C_{B}(M) & \frac{1}{\omega Z_{B_{1}}} \sigma_{B} S_{B}(M) \\
\omega Z_{B_{1}} \zeta_{B} S_{B}(M) & -\frac{\lambda_{B}-\mu_{B}}{2} S_{B}(M)+C_{B}(M)
\end{array}\right),
\end{aligned}
$$

where

$$
S_{A}(N)=\left\{\begin{array}{l}
\frac{\sinh \left(N \theta_{A}\right)}{\sinh \theta_{A}} \text { for } \frac{\mu_{A}+\lambda_{A}}{2}>1 \\
(-1)^{N+1} \frac{\sinh \left(N \theta_{A}\right)}{\sinh \theta_{A}} \text { for } \frac{\mu_{A}+\lambda_{A}}{2}<-1,
\end{array}\right.
$$$$
C_{A}(N)=\left\{\begin{array}{l}
\cosh \left(N \theta_{A}\right) \text { for } \frac{\mu_{A}+\lambda_{A}}{2}>1 \\
(-1)^{N} \cosh \left(N \theta_{A}\right) \text { for } \frac{\mu_{A}+\lambda_{A}}{2}<-1,
\end{array}\right.
$$

$$
\begin{aligned}
& S_{B}(M)=\frac{\sin \left(M \theta_{B}\right)}{\sin \theta_{B}}, \\
& C_{B}(M)=\cos \left(M \theta_{B}\right),
\end{aligned}
$$

where $\theta_{A}$ and $\theta_{B}$ are the real quantities defined by Eqs. (28) [or (32)] and (22), respectively. By inserting Eqs. (53) - (58) into Eq. (51), we can obtain the exact expression for $T_{A B A}$ under the condition (52). (See Appendix A.) The transmission rate is calculated in the same manner as given in Sec. II. The result for the present case $\left(Z_{x}=Z_{y}, Z_{A_{1}}=Z_{B_{1}}\right.$, and $\left.Z_{A_{2}}=Z_{B_{2}}\right)$ is

$$
T=\frac{4}{P^{2}+Q^{2}},
$$

where

$$
P=G_{ \pm} e^{2 N \theta_{A}}+G_{\mp} e^{-2 N \theta_{A}},
$$
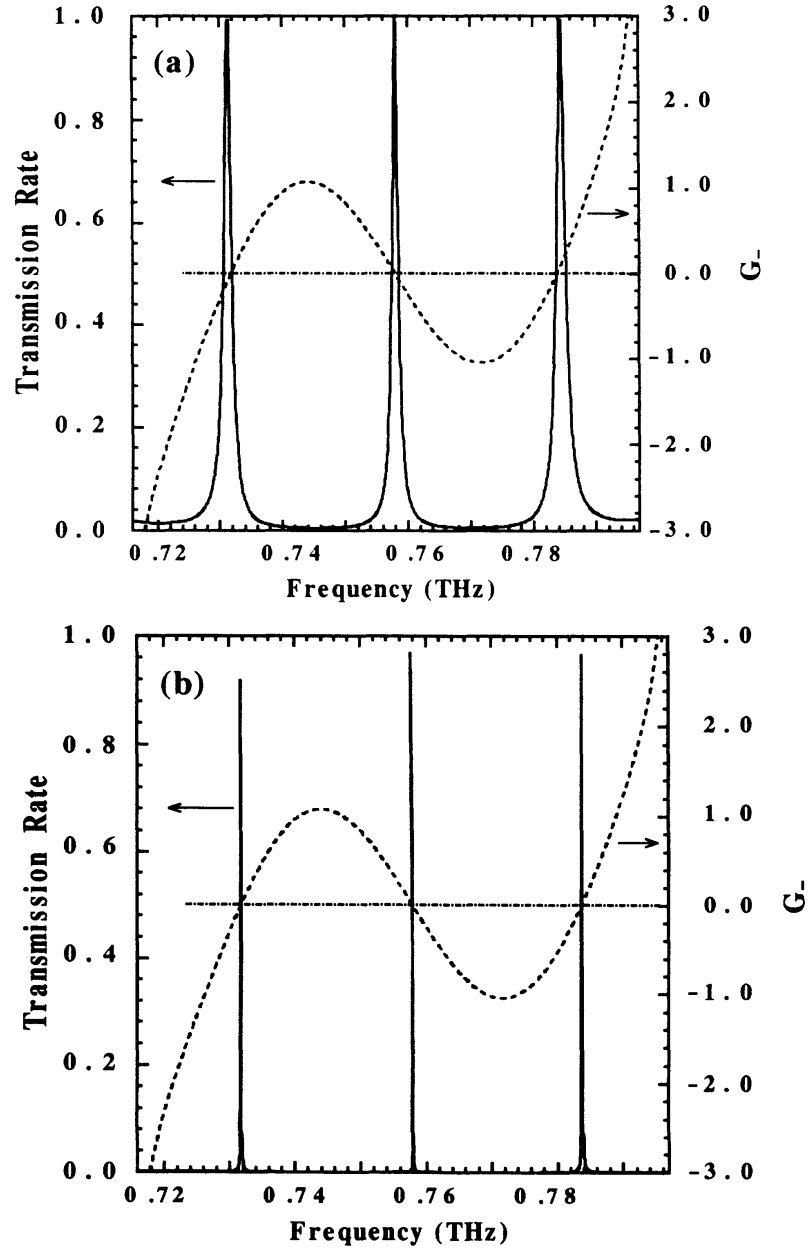

FIG. 5. The L-phonon transmission rate (solid line) and the function $G_{-}$(dotted line) within the $A$-stop band (at the lowest frequencies) in the $A B A$ triple superlattice: (a) $N=10, M=15$; (b) $N=20, M=15$. 


$$
\begin{aligned}
Q= & \pm \frac{\widetilde{\sigma}_{A}-\widetilde{\zeta}_{A}}{2 \sinh \theta_{A}}\left\{G_{ \pm} e^{2 N \theta_{A}}-G_{\mp} e^{-2 N \theta_{A}}\right\} \\
& +\left[\left(\widetilde{\sigma}_{B}-\widetilde{\zeta}_{B}\right)-\frac{\widetilde{\sigma}_{A}-\widetilde{\zeta}_{A}}{2 \sinh ^{2} \theta_{A}} D\right] S_{B}(M), \\
D= & \frac{\left(\lambda_{A}-\mu_{A}\right)\left(\lambda_{B}-\mu_{B}\right)}{2}+\zeta_{A} \sigma_{B}+\zeta_{B} \sigma_{A}, \\
G_{ \pm} & =C_{B}(M) \pm \frac{S_{B}(M)}{2 \sinh \theta_{A}} D .
\end{aligned}
$$

Here, $\widetilde{\sigma}_{A}=\left(Z_{y} / Z_{A_{1}}\right) \sigma_{A}, \widetilde{\zeta}_{A}=\left(Z_{A_{1}} / Z_{x}\right) \xi_{A}, \widetilde{\sigma}_{B}=\left(Z_{y} /\right.$ $\left.Z_{B_{1}}\right) \sigma_{B}$, and $\widetilde{\zeta}_{B}=\left(Z_{B_{1}} / Z_{x}\right) \xi_{B}$; the upper sign corresponds to the case where $\left(\mu_{A}+\lambda_{A}\right) / 2>1$ and the lower sign to $\left(\mu_{A}+\lambda_{A}\right) / 2<-1$. The expression for the transmission in the general case $\left(Z_{x} \neq Z_{y}, Z_{A_{1}} \neq Z_{B_{1}}\right.$, and $\left.Z_{A_{2}} \neq Z_{B_{2}}\right)$ is presented in Appendix $B$. Hence, as the number $N$ of the $A$-superlattice period becomes larger, both $P$ and $Q$ increase exponentially, and therefore the transmission rate becomes zero in proportion to $e^{-4 N \theta_{A}}$. This is valid if the coefficients $G_{ \pm}$of $e^{2 N \theta_{A}}$ in Eqs. (60) and (61) are nonzero. However, if these coefficients are zero, that is

$$
\begin{aligned}
& G_{+}=0 \text { for } \frac{\mu_{A}+\lambda_{A}}{2}>1, \\
& G_{-}=0 \text { for } \frac{\mu_{A}+\lambda_{A}}{2}<-1,
\end{aligned}
$$

is satisfied, the transmission rate has a finite value

$$
T=4\left[\left(\widetilde{\sigma}_{B}-\widetilde{\zeta}_{B}-\frac{\widetilde{\sigma}_{A}-\widetilde{\zeta}_{A}}{2 \sinh ^{2} \theta_{A}} D\right) S_{B}(M)\right]^{-2},
$$

for a large $N$. Therefore, Eq. (64) should be called the resonance condition in the $A B A$ multisuperlattice structures. ${ }^{8}$ In Fig. 5(a), we show the frequency dependence of $G_{-}$(relevant in the frequency range shown in Fig. 4). As expected, we can find a good coincidence of the frequencies at the sharp enhancements in transmission with the frequencies satisfying $G_{-}=0$. The small deviation between frequency satisfying $G_{-}=0$ and the location of the transmission peak arises from the finite number $(N=10)$ of the period assumed for superlattice $A$; that is, the terms of $e^{-2 N \theta_{A}}$ in Eqs. (60) and (61) have small but nonvanishing contributions. ${ }^{9}$ If $N$ becomes larger, the deviation becomes zero. In Fig. 5(b), we show the same plot as Fig. 5(a) for $N=20$. In this figure, the frequencies at the transmission peaks are in excellent agreement with the zero's of $G_{-}$.

As shown in Sec. II, a single periodic superlattice acts as a filter of phonons in stop bands. However, we find that the phonons in the $A$ stop bands can be transmitted through the $A B A$ system when they satisfy the resonance condition (64), even if the period of the $\boldsymbol{A}$ superlattice is infinity.

\section{SUMMARY AND CONCLUDING REMARKS}

In this study, we derived the analytic expressions for the products of transfer matrices and the transmission rate in the periodic superlattice with a finite number of periods. The transmission rate based on our formula coincides, of course, with that calculated by multiplying the transfer matrices for a unit period successively by a computer. We also have applied these exact expressions to the phonon propagation in the $A B A$ multiplesuperlattice system, and discussed the origin of the sharp enhancements in transmission. We have showed that the phonons in the stop bands of the $A$ superlattice can be transmitted through this system (with transmission rate close to unity) when they satisfy the resonance condition (even if the period of the $A$ superlattice is infinity), otherwise the transmission rate vanishes exponentially with the size of the $A$ superlattice.

The results of the present work suggest the possibility of designing phonon optics devices such as a phonon filter, phonon mirror, and phonon resonator. In the periodic superlattice systems [Fig. 2(a)], we can also modulate the phonon frequency of the forbidden gap by changing the thickness of constituent layers. We can also modulate the width of phonon stop bands by changing the constituent materials, i.e., changing acoustic mismatch between constituent layers, or by combining different superlattices. The sharp resonant transmission of phonons in a wide stop band will be used to generate or detect monochromatic high-frequency phonons excited thermally in a crystal at low ambient temperatures. The expressions for the transfer matrix and the transmission rate we derived in the present study will be useful to get important insights into these future applications.

\section{ACKNOWLEDGMENTS}

This work was supported in part by a Research Aid of Inoue Foundation for Science and also by the Suhara Memorial Foundation.

\section{APPENDIX A}

The elements of the transfer matrix $\underline{T}_{A B A}$ [Eq. (51)] under condition (52) are calculated as

$$
\begin{aligned}
\left(\underline{T}_{A B A}\right)_{11}= & \frac{1}{2}\left[1 \pm \frac{\lambda_{A}-\mu_{A}}{2 \sinh \theta_{A}}\right) G_{ \pm} e^{2 N \theta_{A}} \\
& +\frac{1}{2}\left[1 \mp \frac{\lambda_{A}-\mu_{A}}{2 \sinh \theta_{A}}\right] G_{\mp} e^{-2 N \theta_{A}} \\
& \left.+\frac{1}{2}\left[\lambda_{B}-\mu_{B}-\frac{\lambda_{A}-\mu_{A}}{2 \sinh ^{2} \theta_{A}}\right]\right) S_{B}(M), \\
\left(\underline{T}_{A B A}\right)_{12}= & \pm \frac{\sigma_{A}}{2 \sinh \theta_{A}}\left(G_{ \pm} e^{2 N \theta_{A}}-G_{\mp} e^{\left.-2 N \theta_{A}\right)}\right. \\
& \left.+\int \sigma_{B}-\frac{\sigma_{A}}{2 \sinh ^{2} \theta_{A}} D\right] S_{B}(M),
\end{aligned}
$$




$$
\begin{aligned}
\left(\underline{T}_{A B A}\right)_{21}= & \pm \frac{\zeta_{A}}{2 \sinh \theta_{A}}\left(G_{ \pm} e^{2 N \theta_{A}}-G_{\mp} e^{-2 N \theta_{A}}\right) \\
& +\left[\zeta_{B}-\frac{\zeta_{A}}{2 \sinh ^{2} \theta_{A}} D\right] S_{B}(M) \\
\left(\underline{T}_{A B A}\right)_{22}= & \frac{1}{2}\left[1 \mp \frac{\lambda_{A}-\mu_{A}}{2 \sinh \theta_{A}}\right] G_{ \pm} e^{2 N \theta_{A}} \\
& +\frac{1}{2}\left[1 \pm \frac{\lambda_{A}-\mu_{A}}{2 \sinh \theta_{A}}\right] G_{\mp} e^{-2 N \theta_{A}} \\
& -\frac{1}{2}\left[\lambda_{B}-\mu_{B}-\frac{\lambda_{A}-\mu_{A}}{2 \sinh ^{2} \theta_{A}}\right] S_{B}(M)
\end{aligned}
$$

where $G_{ \pm}$and $D$ are defined in Eqs. (63) and (62), respectively.

\section{APPENDIX B}

In the general case $\left(Z_{x} \neq Z_{y}, \quad Z_{A_{1}} \neq Z_{B_{1}}, \quad\right.$ and $Z_{A_{2}} \neq Z_{B_{2}}$ ), the expression of the transmission rate [Eqs. (59) $-(62)$ ] are rewritten as

$$
T=\frac{4\left(Z_{y} / Z_{x}\right)}{P^{2}+Q^{2}}
$$

where

$$
\begin{aligned}
P= & \frac{1}{2} G_{ \pm}\left[1+\frac{Z_{y}}{Z_{x}} \pm \frac{\lambda_{A}-\mu_{A}}{2 \sinh \theta_{A}}\left(\frac{Z_{y}}{Z_{x}}-1\right]\right] e^{2 N \theta_{A}} \\
& +\frac{1}{2} G_{\mp}\left[1+\frac{Z_{y}}{Z_{x}} \mp \frac{\lambda_{A}-\mu_{A}}{2 \sinh \theta_{A}}\left(\frac{Z_{y}}{Z_{x}}-1\right]\right] e^{-2 N \theta_{A}},
\end{aligned}
$$

$$
\begin{aligned}
Q= & \frac{\frac{Z_{y}}{Z_{A_{1}}} \sigma_{A}-\frac{Z_{A_{1}}}{Z_{x}} \zeta_{A}}{2 \sinh \theta_{A}}\left(G_{ \pm} e^{2 N \theta_{A}}-G_{\mp} e^{-2 N \theta_{A}}\right) \\
+ & {\left[\begin{array}{l}
\frac{Z_{y}}{Z_{B_{1}}} \sigma_{B}-\frac{Z_{B_{1}}}{Z_{x}} \zeta_{B} \\
\frac{Z_{y}}{Z_{A_{1}}} \sigma_{A}-\frac{Z_{A_{1}}}{Z_{x}} \zeta_{A} \\
2 \sinh ^{2} \theta_{A}
\end{array}\right] S_{B}(M) }
\end{aligned}
$$

$$
D=\frac{\left(\lambda_{A}-\mu_{A}\right)\left(\lambda_{B}-\mu_{B}\right)}{2}+\frac{Z_{A_{1}}}{Z_{B_{1}}} \zeta_{A} \sigma_{B}+\frac{Z_{B_{1}}}{Z_{A_{1}}} \zeta_{B} \sigma_{A}
$$

Further, the transmission rate $(65)$ at the resonance frequencies are rewritten as

$$
\begin{aligned}
& T=4 \frac{Z_{y}}{Z_{x}}\left[\int \frac{Z_{y}}{Z_{B_{1}}} \sigma_{B}-\frac{Z_{B_{1}}}{Z_{x}} \zeta_{B}\right. \\
& \left.\left.-\frac{\frac{Z_{y}}{Z_{A_{1}}} \sigma_{A}-\frac{Z_{A_{1}}}{Z_{x}} \zeta_{A}}{2 \sinh ^{2} \theta_{A}} D\right] S_{B}(M)\right]^{-2} .
\end{aligned}
$$

${ }^{1}$ For a recent review, see S. Tamura, in PHONONS 89, edited by S. Hunklinger, W. Ludwig, and G. Weiss (World Scientific, Singapore, 1989), p. 703.

${ }^{2}$ V. Narayanamurti, H. L. Stormer, M. A. Chin, A. C. Gossard, and W. Wiegmann, Phys. Rev. Lett. 43, 2012 (1979); O. Koblinger, J. Mebert, E. Dittrich, S. Dottinger, W. Eisenmenger, P. V. Santos, and L. Ley, Phys. Rev. B 35, 9372 (1987); D. C. Hurley, S. Tamura, J. P. Wolfe, and H. Morkoc, Phys. Rev. Lett. 58, 2446 (1987); D. C. Hurley, S. Tamura, J. P. Wolfe, K. Ploog, and J. Nagle, Phys. Rev. B 37, 8829 (1988).

${ }^{3}$ A. S. Barker, Jr., J. L. Merz, and A. C. Gossard, Phys. Rev. B 17, 3181 (1978).

${ }^{4}$ M. Lax and V. Narayanamurti, Phys. Rev. B 24, 4692 (1981).

${ }^{5}$ S. Tamura, Phys. Rev. B 43, 12646 (1991).

${ }^{6}$ L. L. Chang, L. Esaki, and R. Tsu, Appl. Phys. Lett. 24, 593 (1974). For a review, see F. Capasso, K. Mohammed, and A.
Y. Cho, IEEE J. Quantum Electron. QE-22, 1853 (1986).

${ }^{7}$ S. Tamura, D. C. Hurley, and J. P. Wolfe, Phys. Rev. B 38, 1427 (1988).

${ }^{8}$ In the limit of small acoustic mismatch between the constituent layers in superlattice $A$, Eq. (64) becomes $C_{B}(M)=0$. This means that superlattice $B$ is a quarter-wave layer as a whole and Eq. (65) leads to $T=1$ in this limit.

${ }^{9}$ Here we note that the following equation holds at a frequency in the $A$ stop band (the first-order Bragg reflection) in Fig. 2(a):

$$
-\cosh \theta_{A}=\cos \left(\alpha_{1}+\alpha_{2}\right)-\frac{1}{2} \delta^{2} \sin \alpha_{1} \sin \alpha_{2},
$$

where $\delta^{2}=\left(Z_{A_{1}}-Z_{A_{2}}\right)^{2} / Z_{A_{1}} Z_{A_{2}}$. Because $\delta^{2} \ll 1 \quad\left[\delta^{2}\right.$ $=0.0283$ for the L phonons in the (100) GaAs/AlAs superlattice], $\cosh \theta_{A} \leq 1+\frac{1}{2} \delta^{2}$ or $1<e^{\theta_{A}} \leq 1+(\sqrt{2} / 2) \delta$; i.e., $e^{\theta_{A}}$ is close to unity. 